Editorial

\title{
Acknowledgement to Reviewers of the Journal of Functional Biomaterials in 2014
}

JFB Editorial Office, MDPI AG, Klybeckstrasse 64, CH-4057 Basel, Switzerland

Published: 9 January 2015

The editors of the Journal of Functional Biomaterials would like to express their sincere gratitude to the following reviewers for assessing manuscripts in 2014:

\begin{tabular}{lll} 
Alvarez-Lorenzo, Carmen & Hutmacher, Dietmar & Peyton, Shelly \\
Bäumler, Hans & Isbister, James P. & Pittman, Roland \\
Bencharit, Sompop & Jameson, Geoff & Qian, Mingxing \\
Berron, Brad J. & Jayakumar, Rangasamy & Reale, Marcella \\
Cairrão, Elisa & Jonkheijm, Pascal & Rimondini, Lia \\
Caramella, Carla & Kerkhoff, Claus & Romani, Andrea M. P. \\
Dixon, David A. & Kietzmann, Thomas & Sakai, Hiromi \\
Do, Sun Hee & Kwon, Il-Keun & Serda, Rita \\
Dziubla, Thomas & Lammi, Mikko & Shigdar, Sarah \\
Frisch, Benoit & Leipzig, Nic D. & Simoni, Jan \\
Gentile, Piergiorgio & Linninger, Andreas A. & Singh, Arvind \\
Georgieva, Radostina & Liu, Johnson & Song, Jinhua \\
Gloria, Antonio & Lou, Ching-Wen & Soria, Bernat \\
Godin, Biana & Malde, Alpesh & Ullah, Aman \\
Gotor-Fernández, Vicente & Matsusaki, Michiya & Upadhyay, Arun Kumar \\
Harkin, Damien & Mehta, Geeta & Velasco Ortega, Eugenio \\
Hashimotoa, Takashi & Morales, Juan C. & Vergara, Alessandro \\
Hernandez-Montelongo, J. & Müller, W. E. G. & Wang, Qun \\
Ho, Sunita P. & Nagamine, Takeaki & Wennerber, Ann \\
Hong, Yi & Naviglio, Silvio & Woo, Savio L-Y. \\
Huang, Wei Min & Parenteau-Bareil, Rémi & Yager, Dorne \\
& & \\
\hline & &
\end{tabular}


Yoon, Eui-Sung

Yuan, Hanna S.
Zelikin, Alex

Ziebarth, Noël M.

(C) 2015 by the authors; licensee MDPI, Basel, Switzerland. This article is an open access article distributed under the terms and conditions of the Creative Commons Attribution license (http://creativecommons.org/licenses/by/4.0/). 